\title{
Renal allograft aspergilloma after renal transplantation treated with antifungal therapy with a favorable outcome: a case report.
}

\author{
MOHAMMED ALSAEED ${ }^{1}$, Kiran Kalam ${ }^{1}$, and ABEER ALBADANI ${ }^{1}$ \\ ${ }^{1}$ Prince Sultan Military Medical City
}

May 12, 2021

\begin{abstract}
Renal allograft aspergillus is a rare entity with a high mortality due to life-threatening complications. We report a case of renal aspergilloma causing obstructive uropathy after two months of transplantation in a 64 -year- old patient who was managed successfully with antifungal therapy without surgical intervention.
\end{abstract}

Title: Renal allograft aspergilloma after renal transplantation treated with antifungal therapy with a favorable outcome: a case report.

Authors:

1. ABEER AL-BAADANI, MD; Department of Medicine, Infectious Disease Division, Prince Sultan Military Medical City, Riyadh, Saudi Arabia.

2. MOHAMMED ALSAEED, MD; Department of Medicine, Infectious Disease Division, Prince Sultan Military Medical City, Riyadh, Saudi Arabia.

3. Kiran Kalam, MD; Department of Medicine, Infectious Disease Division, Prince Sultan Military Medical City, Riyadh, Saudi Arabia.

\section{Correspondence:}

MOHAMMED ALSAEED, MD

Department of Medicine, Infectious Disease Division, Prince Sultan Military Medical City Makkah Al Mukarramah Rd, As Sulimaniyah, Riyadh 12233, Saudi Arabia.

Email: mohalsaeed@live.com

Phone: +966506273469

Acknowledgements: None.

Sources of support: None.

Disclosures of potential conflicts of interest: None.

\section{Abstract:}

Renal allograft aspergillus is a rare entity with a high mortality due to life-threatening complications in forms of disseminated infections, aneurysmal rupture and bleeding. Immediate recognition with a prompt surgical and medical intervention is strongly advocated. We report a case of renal aspergilloma causing obstructive uropathy after two months of transplantation in a 64 -year- old patient who was managed successfully with antifungal therapy without surgical intervention. Up to our knowledge this is the first case of early localized renal aspergilloma post renal transplant which was uneventfully treated by medical therapy alone. 
Key Words: Kidney Transplant, Aspergillosis.

\section{Case presentation:}

A 64 years old male patient who's known to have uncontrolled diabeties, hypertension, Ischemic heart disease and end stage renal disease; underwent a non related renal transplant in August 2018 in Pakistan. Two months post- transplant the patient developed oozing from his surgical site and deterioration in his graft function for which he was admitted for investigations. One day into admission, he complained of decrease urine output with further rise in serum creatinine. Urgent ultrasound was done which showed dilated pelvicalyceal system .Foleys catheter was inserted which led to improve the urine output. Notably, whitish debris was noticed in his urine .Upon patient 's wish Foley catheter was removed subsequently he developed decrease urine output along with more deterioration of renal function for which Foleys was reinserted but this time renal functions did not improve which necessitated urgent percutaneous nephrostomy on the same day. The patient underwent cystoscopy which revealed whitish colored fluffy ball like structure obstructing the urethra which was retrieved and sent for culture and histopathology (Figure1). The Urine culture and PCR were positive for Aspergillus species. The histopathology showed septated fungal hyphae morphologically consistent with Aspergillus. Of note, serum Galactomannan remained normal through the course ranging from $0.2-0.1$ units. Immunosuppression was stopped, liposomal amphotericin was initially started then switched to voriconazole IV after obtaining the microbiological results. Further, nephrogram showed obstruction at mid distal ureter downwards (figure 2). Cystoscopy showed fungal ball in middle calyx and urinary bladder, which was subsequently washed out.

Despite starting the patient on voriconazole and decreasing his immuno-suppression, his graft function took a long time to improve which could be partly due to possible graft rejection. Patient was offered graft nephrectomy which he declined. Patient was continued on oral voriconazole $200 \mathrm{mg}$ BID.

During in the hospital course his surgical site developed infection secondary to coagulase negative staphylococci. Abdomen CT scan showed thickening of the lower right anterior abdominal wall with subcutaneous fat stranding and multiple air foci, with overlying skin defect. Perinephric fat stranding noted around the transplanted kidney in the right lower quadrant with extension along the transplant ureter. No perinephric fluid collections.

Patient was started on antibiotics and continued on voriconazole until he was discharged successfully .In the Outpatient department he was subsequently started on Mycophenolic acid and tacrolimus .He continued voriconazole for 9 months. Clinically he stayed well with no systemic signs and symptoms ,his renal function returned to baseline of 80 microliter.

\section{Discussion:}

This patient had presented with clinical feature consistent with obstructive uropathy and acute deterioration of renal profile secondary to renal pelvis aspergillus ball as it was evident by the culture and the PCR. Owing to his early presentation after transplantation and the lack of the donor records, we believe it is a donor driven fungal infection. Isolated renal aspergillus has been described after renal transplant with unfavorable graft prognosis specially if it is an early presentation which mostly lead to allograft nephrectomy ${ }^{1}$. Clinically they presented with fever and decrease in the urine output ${ }^{2-5}$. Yet, our patient mainly had decrease in the urine output and increase in the renal profile. More than one possibility could have led to acquire aspergillus as reported previously either from the donor source, contaminated procedures or from the urinary tract infections. The rarity and the high fatality rate of the disease has resulted in a limited medical option for the cure. Thus, the majority consider surgical intervention as an urgent and lifesaving treatment modality owing to the high rate of medical treatment failure and to the angioinvasion with occasional aneurysmal formation ${ }^{2,6}$. Some of the medical treatment which was used amphotericin or liposomal amphotericin itraconazole and voriconazole as a combination or monotherapy ${ }^{2-5,7}$. Fortunately, Our patient has improved on nephrostomy drainage and antifungal liposomal amphotericin followed by voriconazole for a total duration of one year with a good clinical and radiological response. H. Vuruskan etl had reported Similar case of distal ureteric obstruction secondary to aspergilloma which was treated with 
nephrostomy drainage and antifungal However, the patient died after forty days of transplantation due to acute circulatory failure ${ }^{6}$. On the other hand, there are reported cases which were treated by nephrostomy drainage and anti-fungal with a good preserved allograft functions ${ }^{8,9}$. Another report from Shannon et al of late presentation of renal allograft aspergilloma which have been successfully treated medically ${ }^{10}$. Indefinite antifungal therapy would be the preferable approach in non-surgically managed patients as the recurrence pseudoaneurysm was reported with two weeks course of itraconazole but not with three months duration $8,10,11$. Sadagah etl from Saudi Arabia has reported renal allograft aspergilloma who failed the antifungal therapy and ended by nephrectomy ${ }^{12}$.

\section{References:}

1. Fadel FI, Salah DM, Bazaraa HM. Localized Renal Graft Aspergillosis in a Child after Kidney Transplantation: Case Report and Review of Literature. Virol Mycol. 2016;05(03).

2. Garrido J, Lerma JL, Heras M et al. Pseudoaneurysm of the iliac artery secondary to Aspergillus infection in two recipients of kidney transplants from the same donor. Am J Kidney Dis. 2003;41: $488 \mathrm{e} 92$.

3. Liu K-Y, Tsai P-J, King K-L et al. . Pseudoaneurysm of the iliac artery secondary to Aspergillus infection after kidney trans- plantation. J Chin Med Assoc. 2009;72:654e6.

4. Minz M, Sharma A, Kumar S et al. Use of autogenous in- ternal iliac artery for bridging the external iliac artery after excision of Aspergillus mycotic aneurysm in renal transplant recipients. J Vasc Surg. 2011;53:802e4.

5. Ram RC, Ram R, Swarnalatha G et al. 0. True" mycotic aneurysm of the anastomotic site of the renal allograft artery. "Exp Clin Transpl. 2012;10:398e402.

6. Vuruskan H, Ersoy A, Girgin NK, Ozturk M, Filiz G, Yavascaoglu I OB. An Ureteric, unusual cause of ureteral obstruction in a renal transplant recipient: aspergilloma Transpl. 2005;37(5):2115-7.

7. Linden E, Restrepo D, Dikman S et al. T. Aspergillus infec- tion limited to renal allograft: case report and review of literature. ransplant Infect Dis. 2006;8:177e81.

8. Linden E, Restrepo D, Dikman S, Murphy B, Huprikar S. Aspergillus infection limited to renal allograft: case report and review of literature. Transpl Infect Dis . 2006;8(3):177-181. doi:10.1111/j.13993062.2006.00134.x

9. Johnston O, Little DM, Hickey D, Conlon PJ. Aspergillus 'fungus ball' within a cadaveric renal transplant graft. Nephrol Dial Transplant . 2004;19(5):1317-1318. doi:10.1093/ndt/gfh168

10. Shannon EM, Reid MJA, Chin-Hong P. Late aspergilloma of a renal allograft without need for operative management: A case report and review of the literature. Transpl Infect Dis. 2016 Apr 1;18(2):261-5.

11. Wang $\mathrm{R}, \mathrm{Wu} \mathrm{J}$, Wang $\mathrm{Y}$ et al. Aspergillus infection limited to the anastomosed artery following renal transplantation: a report of 4 cases. Transpl Infect Dis. 2009;11:363e6.

12. Sadagah L, Alharbi M, Alshomrani M, Almalki A. Renal Allograft Aspergillus Infection Presenting With Obstructive Uropathy: A Case Report. Transplant Proc. 2017 Jan 1;49(1):193-7.

\section{Figures:}

Figure (1) H \&E stain: renal biopsy septated hyphae at 45-degree angle.

Figure (2) Nephrogram of Transplanted Kidney showing obstruction at mid distal ureter onwards, failure of dye to pass.

\section{Hosted file}

Figure 1 Case Report.pdf available at https://authorea.com/users/413499/articles/521810renal-allograft-aspergilloma-after-renal-transplantation-treated-with-antifungaltherapy-with-a-favorable-outcome-a-case-report

\section{Hosted file}

Figure 2 Case Report.pdf available at https://authorea.com/users/413499/articles/521810renal-allograft-aspergilloma-after-renal-transplantation-treated-with-antifungal- 
therapy-with-a-favorable-outcome-a-case-report
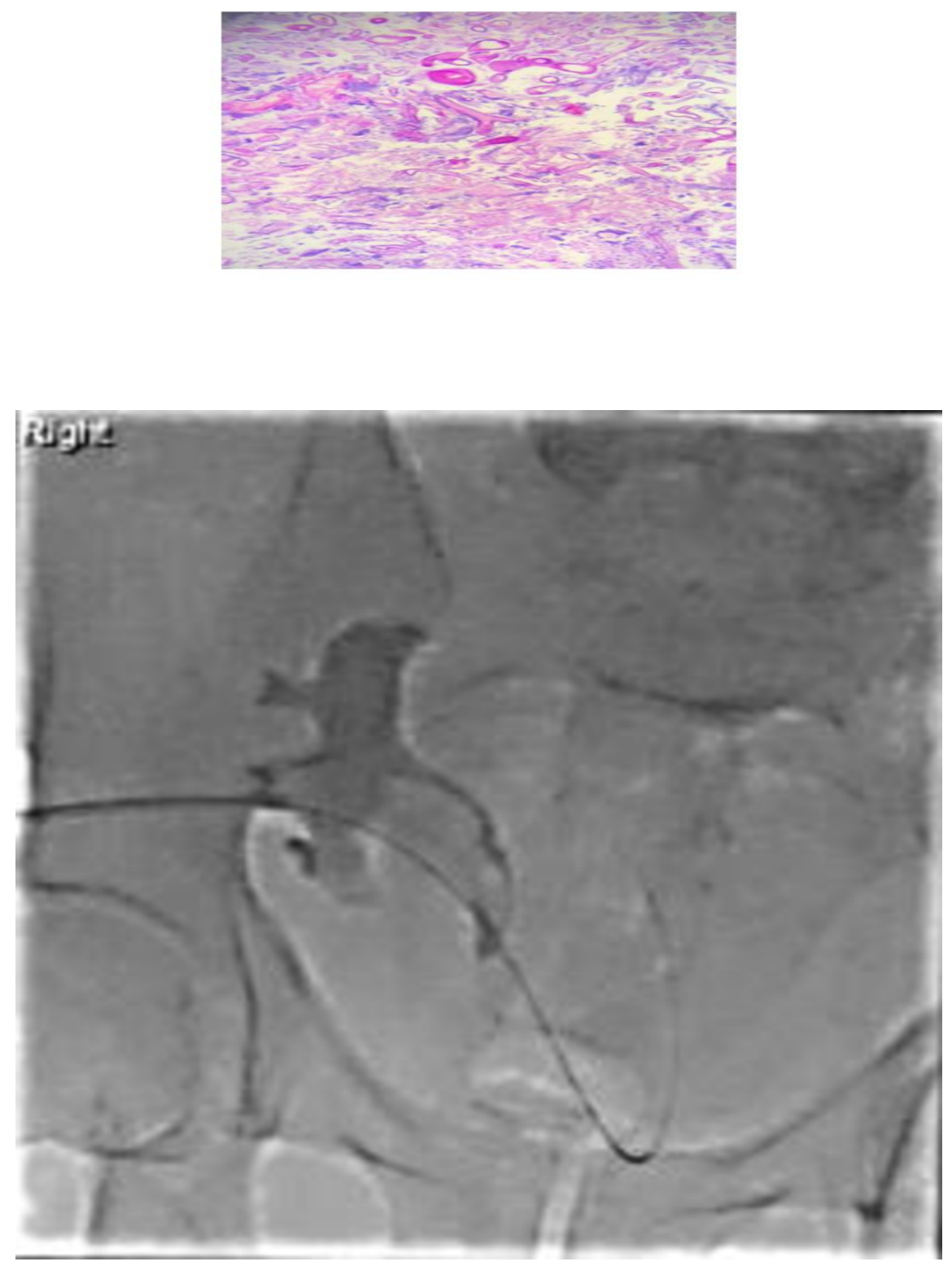